\title{
Counterfactuals and historical possibility
}

Tomasz Placek (uzplacek@cyf-kr.edu.pl)

Department of Philosophy, Jagiellonian University, Grodzka 52, 31-044 Kraków, Poland

Thomas Müller (thomas.mueller@uni-bonn.de)

Philosophisches Seminar, LFB III, Lennéstr. 39, 53113 Bonn, Germany

\begin{abstract}
We show that truth conditions for counterfactuals need not always be given in terms of a vague notion of similarity. To this end, we single out the important class of historical counterfactuals and give formally rigorous truth conditions for these counterfactuals, employing a partial ordering relation called "comparative closeness" that is defined in the framework of branching space-times. Among other applications, we provide a detailed analysis of counterfactuals uttered in the context of lost bets. In an appendix we compare our theory with the branching space-times based reading of counterfactuals recently proposed by Belnap.
\end{abstract}

Keywords: branching space-times, historical counterfactuals, comparative closeness, betting

\section{Introduction}

This paper is a contribution to the analysis of counterfactuals. Like Bennett $(2003,12)$, by a counterfactual we mean a subjunctive conditional with a false antecedent. Typical examples of counterfactuals are the following (it should be a simple matter to supply an appropriate context):

(1) If this coin had shown heads, I would have won my bet.

If this were a ruby, it would be red.

Following Lewis (1973), we will be using " $A \square \longrightarrow C$ " to stand for "If it were the case that $A$, it would be the case that $C$ ". ${ }^{1}$ Antecedent $A$ and consequent $C$ are taken to be declarative sentences in the indicative mood; thus we take the subjunctive mood to be part of the connective.

In our paper, we will be investigating truth conditions for counterfactuals. Some writers, most prominently Dorothy Edgington $(1995,2004)$, have argued that giving truth conditions for any form of conditionals is a misguided project. In this debate we side with Bennett (2003, 253ff.), who argues that even if it makes no sense to give truth conditions for indicative conditionals, giving truth conditions for counterfactuals is a much more reasonable project. 
In giving truth conditions for counterfactuals, it is natural to start with the intuition that one should evaluate the consequent in a scenario that does not deviate much from actuality while making the (actually false) antecedent true. Thus, $A \square \rightarrow C$ is true iff $^{2} C$ is true in the scenario that is most similar to actuality while making $A$ true. This intuition has been spelled out in various ways, e.g., by Stalnaker (1968) and Lewis (1973). ${ }^{3}$ Most authors agree that in a successful analysis the following three notions will play a crucial role: the intuitive concept of "scenario" should be explained in terms of histories (sometimes called just worlds), forks should describe in a localized manner how histories start to deviate, and the evolution of worlds before and after a fork should be smooth. The notion of similarity that is operative in the analysis may be either basic or derivable from these notions.

It is often taken for granted that counterfactuals form a single species and thus, that a single, unified analysis would apply to all of them. That unified analysis is often additionally required to be neutral with respect to a number of controversial issues like determinism vs. indeterminism and the question of whether indexical elements play a role in counterfactuals. The subsequent formal development has shown that if a unified approach is used, the analysis has to be vague. The assumption of uniformity has accordingly been challenged by some writers (e.g., Barker 2003, Bennett 2003, 284ff.). It seems that one can either give a vague, general analysis of counterfactuals or aim at a more rigorous analysis of a subclass of counterfactuals.

In this paper, we will give a rigorous analysis of the class of what we call historical counterfactuals. Such counterfactuals are important in real life situations ("If you had told me the meeting was canceled, I wouldn't have come"), including attributions of agency, praise and blame ("Shame on you: if you hadn't squandered all the money, we would be having a nice dinner now") and singular causation ("If the match hadn't been struck, it wouldn't have lit"). In singling out this class, the key notion is that of real, or historical, possibility. We claim that for the class of historical counterfactuals, formally rigorous truth conditions can be given in the framework of branching space-times.

The paper is structured as follows: In section 2, we will sharpen the distinction between historical and non-historical counterfactuals and explain the concepts involved in the analysis of historical ones. This discussion will also motivate the development of our formal machinery in sections 3-5. Thus, in section 3 , we present the framework of branching space-times, which we use to define a notion of similarity, called "comparative closeness", in section 4 . In section 5 , we specify a formal language whose semantics is based on these developments. In section 6 , we will discuss the merits of our approach. Appendix A considers an 
alternative analysis of counterfactuals in branching space-times, which is due to Nuel Belnap.

\section{Historical and non-historical counterfactuals}

\subsection{Historical Possibility}

In philosophical discussions of modality it is commonly conceded that we can employ a variety of notions of possibility. E.g., there is logical possibility (meaning the absence of contradiction), there are various nomic possibilities (e.g., physical possibility understood as conformity to the laws of physics), etc. We hold that for the analysis of counterfactuals the notion of historical possibility is crucial. ${ }^{4}$

Historical possibilities are everywhere. Consider the fact that you had coffee this morning. (If you didn't, supply a different example.) With respect to this very occasion of your having breakfast, it is true that you could have skipped the coffee. For a different example of the same sort, please move your arm now. Thank you. Certainly you could have not moved your arm on this very occasion.-For examples of a different flavor, consider last week's lottery numbers. At the very occasion on which these numbers were drawn, different numbers could have been drawn. ${ }^{5}$

As our examples show, historical possibilities presuppose indeterminism: real indeterminism of the world we live in, not to be confused with unpredictability, indeterminism of theories or other epistemological notions. We presuppose that some situations can really continue in more than one alternative way. By saying this, we have taken sides in an enormous debate about determinism and indeterminism. Many writers on counterfactuals have assumed that a good theory of counterfactuals must be neutral with regard to this debate, or that it has to presuppose determinism. To us, this appears to be misguided. ${ }^{6}$

In fact, analyses of counterfactuals in the presence of determinism like in Lewis (1973) strike us as involving double talk: The worlds are assumed to be deterministic, but there are so-called "miracles" that are supposed to produce worlds that are minimally different from the actual world while coinciding with actuality in the whole past. Such worlds are ruled out by determinism: assuming determinism, if initial segments of worlds coincide, so do the worlds as wholes. Yet, a notion of (localized) "miracles" is required to make sense of the idea that worlds were alike in their past but later started to deviate. It turns out that Lewisian "miracles" have exactly the same formal structure as the indeterminism in the branching space-times models that we will employ, being localized at single space-time points. ${ }^{7}$

cfsynth_revised_18mar05.tex; 20/04/2005; 12:42; p.3 


\subsection{Counterfactuals And historical Possibility}

Some counterfactuals clearly involve historical possibility, and some clearly do not. E.g., the betting example above (1) appeals to historical possibility. To determine its truth value in a given scenario, one has to consider whether in the closest historically possible scenario in which the antecedent holds, i.e., in which the coin landed heads, I won my bet. Thus, if in fact I bet on heads and the coin that was tossed could have shown heads, ${ }^{8}$ the counterfactual will be true. If no such historical possibility exists, we do not know what to say. Things are different in the ruby example (2): If something is not a ruby, then there is no historical possibility of that very thing's being a ruby. ${ }^{9}$ However, in evaluating counterfactual (2), historical possibilities are not an issue: the counterfactual is evaluated in what Bennett $(2003,284)$ calls the "direct" manner, merely as an instance of the generalization "All rubies are red". We share Bennett's diagnosis that "direct" counterfactuals, which we prefer to call "non-historical", are "uninteresting-because unchallenging - as a philosophical topic" $(2003,287) .{ }^{10}$

We also agree that some sentences may be interpreted as historical or non-historical counterfactuals, depending on context. To use Bennett's example (2003, 285), consider the following:

If Charles had been CEO of Enron, the accounting fraud would not have lasted a week.

This sentence will normally be interpreted non-historically, describing the actual financial abilities of Charles: He is too inept at handling money to invent and sustain something as intricate as the Enron fraud. The counterfactual will be true if and only if Charles is in fact financially inept. However, the sentence may also be interpreted historically, taking us back to a time in the past when Charles might have chosen a different career. On this reading, Charles's current financial inability is not relevant - whether (3) is true depends on the existence of historical possibilities through which Charles would have become the CEO of Enron and on the attitude he would have had toward the fraud then.

In fact, it appears to us that borderline cases between historical and non-historical counterfactuals are quite common. Consider, e.g., Lewis's notorious kangaroo sentence,

If kangaroos had no tails, they would topple over.

An historical reading would have to consider evolution, facing difficult questions of species identity, while a non-historical reading might, e.g., point out the importance of tails for the kangaroos' balancing. But even

cfsynth_revised_18mar05.tex; 20/04/2005; 12:42; p.4 
given an abundance of borderline cases, there is a very large amount of clearly historical counterfactuals.

\subsection{Historical COUNTERFACTUALS AT WORK}

In order to illustrate the uses of historical counterfactuals, we start by slowly unrolling the betting example (1) from above. We will encounter some problems along the way. Solving these problems is the main objective of the formal theory that we develop below.

Our betting example is the following: $U$ bet on heads and the coin was tossed, showing tails. On seeing the result, $U$ exclaimed: "If the coin had shown heads, I would have won my bet". In this case, intuitions are quite clear: The counterfactual is true. Why is that so?

At the event of utterance $e$, the antecedent $A$ ("the coin showed heads") is false, and so is the consequent $C$ ("I won my bet"). The utterance is a conditional in the subjunctive mood with a false antecedent, so it qualifies as a counterfactual according to our definition. Both antecedent and consequent are sentences in the past tense that are evaluated at $e$, thus indexically referring to events earlier than $e$. Apart from tense, a further source of indexicality in the example is the use of the pronoun "I" in the consequent. Since the counterfactual is clearly not an instance of a generalization, it has to be evaluated historically. Which histories need to be considered? There is the actual history up to $e$, which is fixed by the event of utterance, $e$, and which comprises $e$ 's actual past (in which, to repeat, both the antecedent and the consequent are false). ${ }^{11}$ Apart from the actual history up to $e$, we need to consider other histories in which the antecedent $A$ is true (socalled $A$-histories). There is a history in which everything was as it actually was, except for minute details of the tossing which led to the coin's showing heads. There is also a history in which the coin landed heads and additionally, while the coin was up in the air, I changed my bet from heads to tails, and there are many other histories making the antecedent true. Now the intuitive recipe for assessing the truth value of our counterfactual is this: $A \square \longrightarrow C$ is true just in case at the $A$-history that is closest to actuality, $C$ is true. Intuitively, the closest $A$-history is the one we mentioned first, involving only the minimal changes required to make $A$ true. Since in this world, $U$ still bet on heads and the coin landed heads, $U$ won her bet, making $C$ and thus, the counterfactual, come out true.

In our description, we have skipped one crucial detail: Since we are dealing with indexical sentences, we need to specify where they are to be evaluated. $A \square \longrightarrow C$ obviously has to be evaluated at $e$, the event of utterance. But where do we evaluate $A$ and $C$ in histories other than 
the actual one? How can we say that a certain history is an $A$-history? After all, $e$ does not belong to the histories that we mentioned. In order to answer the question, we need to specify, for each non-actual history, an event $e^{\prime}$ at which $A$ and $C$ can be evaluated in order to assess the counterfactual. Our guiding idea will be to specify, for each history, a "counterpart" of event $e$ that has the same spatio-temporal location as $e$. Then we will evaluate $A$ and $C$ with respect to these counterparts $e^{\prime}$. Thus, semi-formally, $A \square \longrightarrow C$ is true at $e$ iff at the history closest to actuality at whose $e^{\prime}, A$ is true, $C$ is true at $e^{\prime}$ as well. Apart from making this fully rigorous, further tasks are to clarify what a history is, which notion of forks among histories is appropriate, and how to analyze the intuitive notion of similarity.

In the following three sections we will give our answers to these questions. As a background theory, we will sketch the framework of branching space-times (Belnap 1992). In this indeterministic framework, histories are something like maximally consistent sets of possible point events. Histories branch at points, thus providing a localized notion of forks. The spatio-temporal ordering of these branching points allows us to define a notion of comparative closeness. The notion of "same spatio-temporal location" will be implemented as an order-preserving equivalence relation. Thus, the framework that we will develop allows us to specify a formal semantics for the limited, but important class of historical counterfactuals.

\section{Branching space-times}

\subsection{BASIC BRANCHING SPACE-TIMES}

We will use Belnap's (1992) theory of branching space-times (BST) to build a semantics for historical counterfactuals. BST is a version of a possible worlds theory that combines indeterminism with relativistic space-times. The role of possible worlds is played in BST by histories, which however, in contrast to Lewis's possible worlds, share initial segments. Technically, the point of departure is a partially ordered non-empty set $\mathcal{W}=\langle W, \leqslant\rangle$, from which histories are to be carved. The elements of $W$ are thought of as spatio-temporal point-like events. The relation $a \leqslant b$ is interpreted as meaning that $a$ is in the causal past of $b$, or, equivalently, that $b$ is in a possible future of $a . a<b$ is short for $(a \leqslant b$ and $a \neq b)$. In a tensed language, one may say that $\mathcal{W}$ consists of all (point) events that occurred, or could have occurred, are occurring or might be occurring, and might occur in the future; this licenses calling $\mathcal{W}$ Our World.

cfsynth_revised_18mar05.tex; 20/04/2005; $12: 42 ;$ p.6 
In general, there are two kinds of forks in Our World $\mathcal{W}$ : upward forks characterized by $x<y, x<z$ and $y$ and $z$ incomparable by $\leqslant$, and downward forks characterized by $y<x, z<x$ and $y$ and $z$ incomparable by $\leqslant$. An upward fork can mean one of two things: (1) $y$ and $z$ belong to alternative possible futures of $x$ or (2) $x$ belongs to the common past of causally unconnectable (space-like separated) $y$ and $z$. In contrast, a downward fork has only one sensible reading, namely, causally unconnectable $y$ and $z$ belong to the past of $x$. This intuition, that any three events forming a downward fork belong to a history, motivates the following definition of history. It first defines upward directed subsets of $W$, and secondly identifies histories with maximal upward directed subsets of $W$ :

\section{Definition 1 (upward directed subset and history)}

$A$ is an upward directed subset of $W$ if for any $x, y \in A$ there is a $z \in A$ such that $x \leqslant z$ and $y \leqslant z$. And $h$ is a history in $W$ if $h$ is a maximal upward directed subset of $W$.

In BST models there can be point events that belong to one history, but which are incomparable by the ordering $\leqslant$. Using a term from physics, such events are called space-like separated:

\section{Definition 2 (space-like separation)}

Two point events $a, b$ of $\langle W, \leqslant\rangle$ are space-like separated iff (1) there is a history $h$ in $\mathcal{W}$ such that $a \in h$ and $b \in h$ and (2) neither $a \leqslant b$ nor $b \leqslant a$.

To introduce some notation, Hist stands for the set of histories of $W$, and $H_{[E]} \stackrel{d f}{=}\{h \in H i s t \mid E \subset h\}$. For $E=\{e\}$, the notation $H_{(e)}$ is used. In BST, histories branch, and a relevant notion is undividedness. Intuitively, we think of two histories as undivided at event $e$ provided that the two share some event above $e$.

\section{Definition 3 (undividedness)}

For $g, h \in H_{(e)}, g$ and $h$ are undivided at a point event $e, g \equiv_{e} h$, iff $g$ and $h$ share some point properly above $e$, if there are any.

If two histories containing $e$ are not undivided at a given event $e$, we say they are divided at $e$. BST's postulates then guarantee that (1) undividedness at $e$ is an equivalence relation on the set $H_{(e)}$ of histories containing $e$, and that (2) any two distinct histories are divided at some event. ${ }^{12}$ With respect to the notion of accessibility, which plays a prominent role in other possible-worlds-frameworks, we can thus say that any two histories are accessible one from the other. 
Choice points for two histories are points at which histories divide, i.e., maximal elements in their intersection:

\section{Definition 4 (choice points)}

For histories $g$ and $h$ of $W$, if $g \neq h$ then the set $C_{g h}$ of choice points for histories $g$ and $h$ is the set of maximal elements of $g \cap h$. Otherwise $C_{g h}=\emptyset$.

A particular realization of BST is a Minkowskian branching structure, in which all histories are Minkowski space-times. ${ }^{13}$ Figure 1 illustrates branching of two histories in two cases: (A) with a single choice point and (B) with two choice points. The region of points space-like separated from the choice points belongs to both histories, as convincingly shown by Belnap (1992, 411ff.).

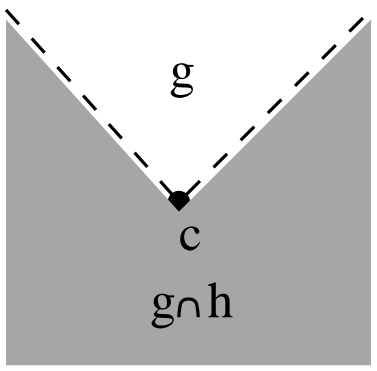

A

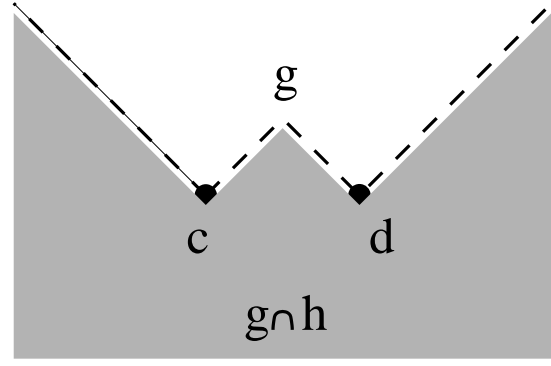

B

Figure 1. (A) Two histories $g$ and $h$ separate at a single choice point $c$. (B) Separation at two choice points $c$ and $d$. The shadowed regions indicate where the histories $g$ and $h$ overlap. The 'surfaces of divergence', represented by broken lines, do not belong to the region of overlap, but the choice points do.

\subsection{BST EXTENDED: LOCATIONS AND PROPERTIES}

As we will show in section 4, basic BST permits one to define comparative closeness of histories via the ordering of their choice points. Yet, the theory is too austere to yield informative counterfactualsappendix A shows what the problems are. Accordingly, we will extend the theory of branching space-times, and will do it in two steps: we will first introduce same locations and then properties.

Same location Recall that events of $\mathcal{W}$ are point-like concrete particulars. As we pointed out, for the assessment of the constituents of a counterfactual we need to be able to say, of two events of $\mathcal{W}$, that 
they occur at the same spatio-temporal location. This can be done in the following way (cf. Müller 2005):

\section{Definition 5 (BST with spatio-temporal locations)}

A triple $\langle W, \leqslant, L\rangle$ is a model of branching space-times with locations if $\langle W, \leqslant\rangle$ is a BST model and $L$ is an equivalence relation on $W$ such that

1. for each history $h$ in $W$ and for each equivalence class $[l], l \in W$, the intersection $h \cap[l]$ contains exactly one element, and

2. $L$ respects the ordering, i.e., for $[l]$ and $\left[l^{\prime}\right]$ equivalence classes and $h_{1}$ and $h_{2}$ histories, $[l] \cap h_{1}=\left[l^{\prime}\right] \cap h_{1}$ iff $[l] \cap h_{2}=\left[l^{\prime}\right] \cap h_{2}$, and the same for $<$ and $>$.

If point-events $x$ and $y$ are members of the same equivalence class $[l]$, we say that they have the same spatio-temporal location.

The construction of a Minkowskian branching structure, mentioned above, guarantees that locations can be introduced. ${ }^{14}$

In what follows, we always assume that we are working with Minkowskian branching structures. Thus, the set of locations, which is the

quotient structure $S \stackrel{d f}{=} W / L$, is Minkowski space-time. This means that (1) we have available vectors connecting any two locations and (2) these vectors can be classified as spacelike, timelike or lightlike in the standard manner.

Properties So far, we can talk about point events and their location, but not about their properties. Still, this will be needed in assessing counterfactuals. ${ }^{15}$ Intuitively, it could be said that in a BST model, properties are already there, since the elements of $W$ are pictured as concrete point-like particulars. Formally, these properties are taken from a set $\mathcal{P}$, and are made available for the semantics of our language in the following way:

\section{Definition 6 (property assignment)}

$A$ property assignment is a function $A: W \rightarrow$ Pow $\mathcal{P}$, where $\mathcal{P}$ is a set of properties.

Thus, each point is assigned a set of properties.

BST-structures that include both locations and property ascriptions are called $\mathrm{BST}+\mathrm{L}+\mathrm{A}$-structures, they are quintuples $\langle W, \leqslant, L, \mathcal{P}, A\rangle$ with $L, \mathcal{P}$, and $A$ as just explained. 


\section{Comparative closeness}

The BST framework makes it possible to define similarity of histories as comparative closeness. The underlying intuition is that histories are the closer the later they branch. ${ }^{16}$ Comparative closeness is thus defined in terms of the locations of choice points.

We will now first give a definition of global comparative closeness, comparing histories without taking into account the event of utterance of a counterfactual. It will turn out that many histories must be treated as incomparable (Section 4.1). In a second step, we will use the event of utterance to define a local notion of comparative closeness (Section 4.2). The formal properties of the closeness ordering will be the same in both cases: we will define families of partial orderings $\angle_{h}$ that can be used in a formal semantics for counterfactuals. ${ }^{17}$

\subsection{Global comparative Closeness}

Given a reference history $h$ and two histories $f$ and $g$ with respective sets of choice points $C_{f h}$ and $C_{g h}$, our task now is to define when the relation $f \angle_{h} g$ holds, i.e., when $f$ is strictly closer to $h$ than $g$. In order to proceed systematically, we reason by cases based on the size of the sets $C_{f h}$ and $C_{g h}$, starting with the '0-0' case where both $C_{f h}$ and $C_{g h}$ have zero elements:

0-0 As $C_{f h}=\emptyset=C_{g h}$, the histories $f=g=h$. Consequently, neither $f{ }_{h} g$ nor $g \angle_{h} f$.

0-1 $C_{f h}=\emptyset$, thus, $f=h$. As $g \neq h$, we have $f{ }_{h} g$.

1-1 Let $C_{f h}=\{c\}, C_{g h}=\{d\}$. We need to distinguish three cases:

(A) $c=d: f$ and $g$ are tied with respect to closeness to $h$, since they split off at the same point. Thus, neither $f L_{h} g$ nor $g L_{h} f$.

(B) $c<d: g \angle_{h} f$, because $g$ branches off from $h$ at $d$, which is later than $c$. (The case $d<c$ is symmetrical.)

(C) $c$ and $d$ are space-like separated: Since $c$ and $d$ are incomparable, the histories $f$ and $g$ are also counted as incomparable with respect to $h$, i.e., neither $f \angle_{h} g$ nor $g \angle_{h} f$.

0-2 As in the 0-1 case, $f \angle_{h} g$.

1-2 Let $C_{f h}=\{c\}, C_{g h}=\left\{d_{1}, d_{2}\right\}$. We need to distinguish six cases, which are illustrated in Figure $2(\mathrm{~A})-(\mathrm{F})$ :

(A) $c=d_{1}: f \angle_{h} g$, since there is a region in $f \cap h$ that is not in $g \cap h .\left(c=d_{2}\right.$ is symmetrical. $)$

cfsynth_revised_18mar05.tex; 20/04/2005; 12:42; p.10 

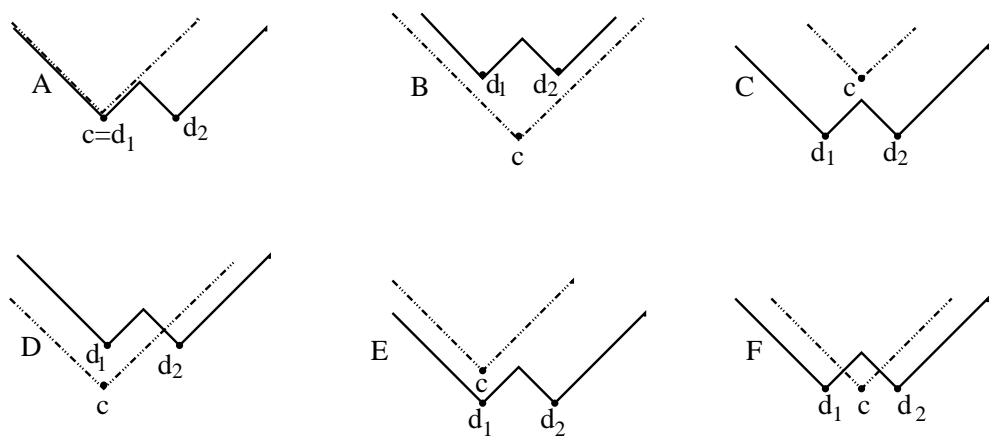

Figure 2. The six subcases to be considered for the 1-2 case.

(B) $c<d_{1}$ and $c<d_{2}: g \angle_{h} f$, since $g$ branches off after $f$.

(C) $d_{1}<c$ and $d_{2}<c$ : $f \angle_{h} g$, since $f$ branches off after $g$.

(D) $c<d_{1}$, and $c$ and $d_{2}$ are space-like separated: Due to the spacelike separation, $f$ and $g$ are incomparable w.r.t. $h$ : neither $f \angle_{h} g$ nor $g \angle_{h} f$ (symmetrically for $d_{2}$ ).

(E) $d_{1}<c$, and $c$ and $d_{2}$ are space-like separated: $f \angle_{h} g$, since $f$ branches off after $g$ (symmetrically for $d_{2}$ ). (C) is a special case of this.

(F) $c$ and $d_{1}$ as well as $c$ and $d_{2}$ are space-like separated: Due to the space-like separation, $f$ and $g$ are incomparable w.r.t. $h$ : neither $f \angle_{h} g$ nor $g \angle_{h} f$.

(The case $d_{1}<c<d_{2}$ cannot occur, since $d_{1}$ and $d_{2}$ are space-like separated.)

A discussion of sets of larger size does not reveal qualitatively new cases. Complete spatio-temporal overlap means tie. If locations of surfaces of divergence do not intersect, it is always clear which history is to count as closer - partial overlap does not change the verdict. If there is intersection, our intuitions do not allow us to count one history as closer than the other.

We thus arrive at the following definition of comparative closeness: ${ }^{18}$

\section{Definition 7 (global comparative closeness)}

Given histories $f, g$, and $h$ and sets of choice points $C_{f h}$ and $C_{g h}, f$ is strictly closer to $h$ than $g\left(f \angle_{h} g\right)$ iff

(1) $C_{f h} \neq C_{g h} \quad$ and $\quad(2) \forall c \in C_{f h} \exists d \in C_{g h} d \leqslant c$.

The first conjunct, $C_{f h} \neq C_{g h}$, prohibits total overlap of locations of surfaces of divergence. The second conjunct prohibits the intersection 
of locations of surfaces of divergence, allowing for partial overlap (cf. the $0-1$ case and $1-2(\mathrm{~A}))$. Notice that already by our decision on the $0-1$ case, we opt for what Lewis calls 'strong centering': history $h$ is always considered closer to itself than any other history.

The global notion of comparative closeness requires one to take a god's eye view on all histories: the ordering is based on all histories in their entirety. Many histories will be incomparable. In an actual utterance of an historical counterfactual, only relatively few histories matter - in fact, only those that branch off in the utterance event's causal past. Thus it makes sense to move from a global notion of comparative closeness to a local notion that considerably narrows the number of histories to be considered.

\subsection{Local COMPARATive ClOSENESS}

For our local notion, we consider an utterance event $e$. The historical alternatives from the point of view of $e$ are exactly those histories that branch off from $e$ 's histories in the causal past of $e$. Thus, for each pair of histories $f$ and $g$ of $\mathcal{W}$ with the set of choice points $C_{f g}$, we define the pruned set of choice points

$$
C_{f g}^{e} \stackrel{d f}{=}\left\{c \in C_{f g} \mid c<e\right\} .
$$

Our definition of local comparative closeness with respect to $e\left(\angle_{h}^{e}\right)$ runs exactly like Definition 7 above:

\section{Definition 8 (local comparative closeness)}

Given an utterance event $e$, histories $f, g$, and $h$ and pruned sets of choice points $C_{f h}^{e}$ and $C_{g h}^{e}, f$ is strictly closer to $h$ than $g\left(f \angle_{h}^{e} g\right)$ iff (1) $C_{f h}^{e} \neq C_{g h}^{e} \quad$ and $\quad(2) \forall c \in C_{f h}^{e} \exists d \in C_{g h}^{e} d \leqslant c$.

\section{The formal language and its semantics}

In the last two sections we developed the resources that can be used to specify truth conditions of counterfactuals in a formal language: We have available $\mathrm{BST}+\mathrm{L}+\mathrm{A}$-structures $\langle W, \leqslant, L, \mathcal{P}, A\rangle$ with $S \stackrel{d f}{=} W / L$ isomorphic to Minkowski space-time, and we may employ the local comparative closeness ordering $\angle_{h}^{e}$. We will now specify the formal language syntactically (section 5.1) and then semantically (section 5.2). 


\subsection{SyntaX}

As we mentioned above, the language we employ is inherently indexical. Sentences will be evaluated at an index of utterance. In line with PriorThomason semantics, the index consists both of an event of utterance $e \in W$ and a history of evaluation $h$ such that $e \in h .^{19}$

(AT) Atomic sentences are propositional constants $p$, to be interpreted as " $p$ here and now". (Thus, properties of events are expressed as propositions true or false at events.) It will be enough to have at most countably many such constants.

Complex sentences are built out of atomic ones by the following means:

(TF) The standard truth-functional connectives.

(S) A family of (one-place) translation operators, taking us from a point of evaluation to a different point: For each vector $s \in S$, we have an operator $[+s]$. (These operators are self-dual.)

(PF) The two (one-place) weak tense operators, $P$ and $F$. (Their strong duals, $H$ and $G$, respectively, are defined as abbreviations: $H \stackrel{d f}{=}$ $\neg P \neg ; G \stackrel{d f}{=} \neg F \neg$.)

(CF) The (two-place) counterfactual conditional, $\square \longrightarrow$.

\subsection{Semantics}

A model $\mathfrak{M}$ is a $\mathrm{BST}+\mathrm{L}+\mathrm{A}$ structure $\langle W, \leqslant, L, \mathcal{P}, A\rangle$ together with an interpretation $I$ that specifies, for each propositional constant $p$, a property $I(p) \in \mathcal{P}$. We evaluate a sentence $\phi$ in a model $\mathfrak{M}$ at index $e, h$, as explained above. The recursive semantic clauses are as follows:

(AT) If $\phi$ is atomic $p$, then $\mathfrak{M}, e, h \models \phi$ iff $I(p) \in A(e)$. I.e., an atomic sentence $p$ is true at $e$ (the history of evaluation, $h$, plays no role here) iff the property $I(p)$ applies to $e$.

(TF) The clauses for the truth-functional connectives are the standard ones.

(S) If $\phi=[+s] \psi, s \in S$, then $\mathfrak{M}, e, h \models \phi$ iff $\mathfrak{M}, e^{\prime}, h \models \psi$, where $e^{\prime}$ is the event in history $h$ whose space-time distance to $e$ is $s$. This spells out the meaning of $[+s]$ as a translation operator. 
(PF) If $\phi=P \psi$, then $\mathfrak{M}, e, h \models \phi$ iff $\mathfrak{M}, e, h \models[+s] \psi$ for some pastdirected timelike or lightlike $s \in S$, i.e., iff there is a translation to an event $e^{\prime}$ in $e^{\prime}$ 's causal past such that at $e^{\prime}, h, \psi$ is true.-The clause for the future operator $F$ is exactly analogous, replacing "past-directed" by "future-directed".

As to the counterfactual conditional, we develop the semantic clause in two steps. In the first step, we temporarily subscribe to what is called the "limit assumption", which says that for any actual scenario and any antecedent sentence, there is a unique closest scenario making the antecedent true. In our framework, this assumption amounts to the following: For each index $e, h$ and for each sentence $\psi_{1}$, there is, with respect to the local comparative closeness ordering $\angle_{h}^{e}$, a unique closest history $h^{\prime}\left(e, h, \psi_{1}\right)$ such that $\psi_{1}$ is true at $e^{\prime}, h^{\prime}$, where $e^{\prime}$ is the event in $h^{\prime}$ that is at the same location as $e$. Given this assumption, our semantic clause looks as follows:

$\left(\mathrm{CF}^{*}\right)$ If $\phi=\psi_{1} \square \rightarrow \psi_{2}$, then $\mathfrak{M}, e, h \models \phi$ iff $\mathfrak{M}, e^{\prime}, h^{\prime} \models \psi_{2}$, where $h^{\prime}=h^{\prime}\left(e, h, \psi_{1}\right)$ is the closest history making $\psi_{1}$ true and $e^{\prime}$ is as just explained.

Since the limit assumption is controversial, we had better not assume it. Our official semantic clause is based on Lewis's (1981) analysis, since the local comparative closeness ordering $\angle_{h}^{e}$ is a partial ordering as presupposed there: ${ }^{20}$

(CF) If $\phi=\psi_{1} \square \rightarrow \psi_{2}$, then $\mathfrak{M}, e, h \models \phi$ iff for any history $h_{1}$ s.t. $\mathfrak{M}, e_{1}, h_{1} \models \psi_{1}$, there is some history $h_{2}$ s.t. $\mathfrak{M}, e_{2}, h_{2} \models \psi_{1}$ and (1) $h_{1}=h_{2}$ or $h_{2} L_{h} h_{1}$, and (2) for any history $h_{3}$ such that $h_{2}=h_{3}$ or $h_{3} \angle_{h} h_{2}$ for which $\mathfrak{M}, e_{3}, h_{3} \models \psi_{1}$, we have $\mathfrak{M}, e_{3}, h_{3} \models \psi_{2}$.- Here, $e_{i}$ is the event in history $h_{i}$ that is at the same location as $e$.

For most practical purposes it will be appropriate to think of the clause $\left(\mathrm{CF}^{*}\right)$ and rest assured that cases in which the limit assumption fails are handled correctly.

A worked-out example Assume that five minutes ago, you looked in the fridge and found no cheese. Now you say to your friend:

If I had found some cheese, I would offer it to you now.

In order to assess this counterfactual, you need to know the modal facts, which are these: In the morning, you deliberated whether to go to the shop and buy some cheese: you could have bought some cheese and put it in the fridge, but you did not. Yesterday, as a matter of fact 
$\mathrm{h}$

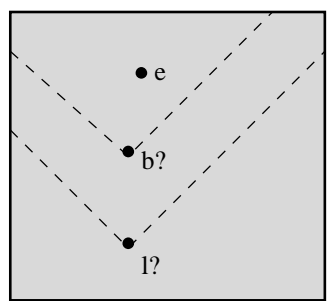

h'

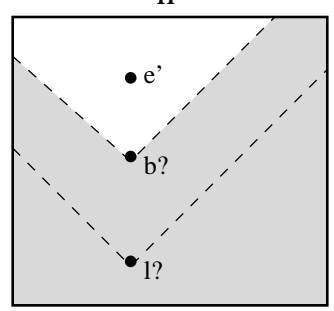

h"

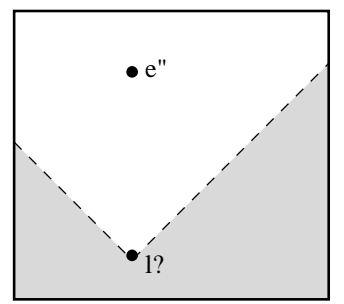

Figure 3. The cheese story. History $h$ with the event $e$ of utterance, choice point $b$ ? (buy the cheese in the morning or not) and choice point $l$ ? (leave the rancid cheese in the fridge or not). The antecedent ("I found some cheese") and the consequent ("I offer it to you now") are true at $e^{\prime}, h^{\prime}$, and $h^{\prime} \angle_{h}^{e} h^{\prime \prime}$. Shadowed regions indicate where $h$ and a given history overlap.

you cleaned the fridge and threw out a piece of rancid cheese, but you could have left the piece where it was. Figure 3 shows the corresponding branching model, which contains three histories. In assessing the counterfactual (5), let us assume that you belong to the amiable class of people who never offer rancid cheese to friends. The counterfactual is evaluated at event $e$ in history $h$. The antecedent is true both at $e^{\prime}, h^{\prime}$ and at $e^{\prime \prime}, h^{\prime \prime}$, but the consequent is only true at $e^{\prime}, h^{\prime}$. The definition of local comparative closeness yields the verdict $h^{\prime} \angle_{h}^{e} h^{\prime \prime}$ : due to the spatiotemporal location of the choice points between histories $h, h^{\prime}$ and $h, h^{\prime \prime}$, respectively, history $h^{\prime}$ (in which you bought fresh cheese this morning) is closer to actuality than history $h^{\prime \prime}$ (in which you found the rancid cheese in your fridge). Thus, the counterfactual is true at $e, h$.

\section{Discussion}

Our formal analysis shows that historical and non-historical counterfactuals need to be treated separately if rigor is wanted at least for some of them. In non-historical cases like the ruby example (2), the resources presupposed in our analysis are simply not available, while in historical cases like the betting example (1), the additional rigor may help to clarify some controversial aspects.

\subsection{A Closer LOOK AT THE BetTing CASE}

Recall the betting example: $U$ bet on heads, the coin showed tails, and the counterfactual utterance, "If the coin had shown heads I would have won my bet", is widely accepted as a true historical counterfactual: In 
the closest history $h^{\prime}$ in which at $e^{\prime} \in h^{\prime}$, the antecedent is true ("the coin showed heads"), the consequent is also true ("I won my bet").

There is, however, a controversy concerning the quite similar counterfactual, uttered in the same circumstances: ${ }^{21}$

If I had bet on tails, I would have won my bet.

Intuitively, most people hold that this is true, assuming that the outcome of the toss is independent of the act of betting. However, many theories of counterfactuals, including Lewis's, give the opposite verdict and hold that the counterfactual is false. Our theory can be employed to diagnose and remove this tension.
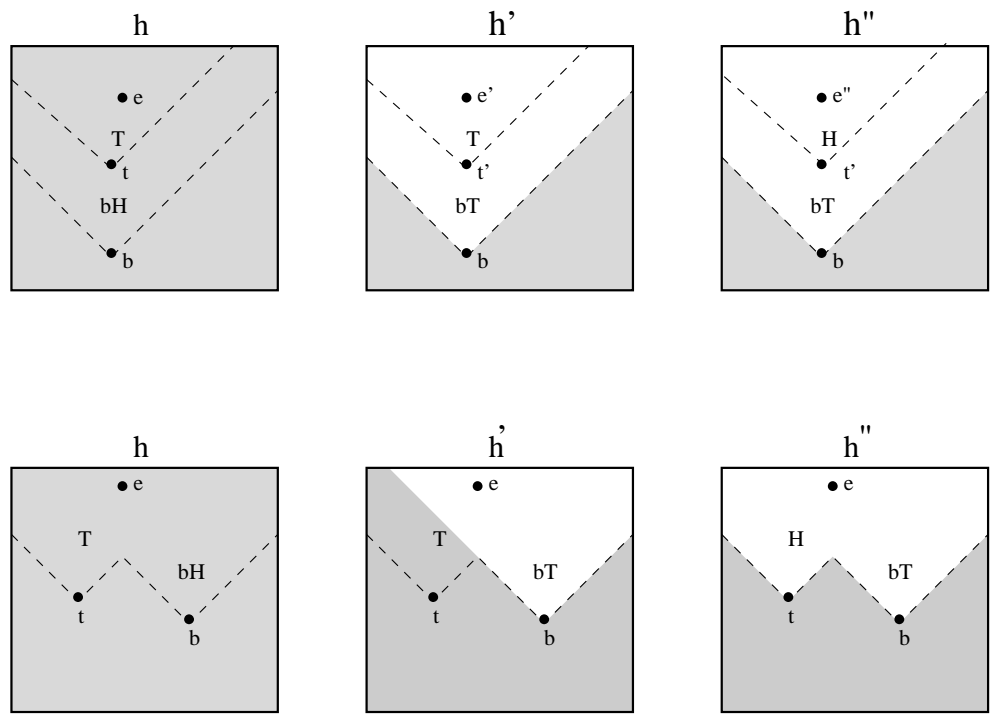

Figure 4. Two models of the betting story. At the top: betting occurs before tossing - $h^{\prime}$ and $h^{\prime \prime}$ are equally close to $h$ and the counterfactual (6) comes out false at $e, h$. At the bottom: betting and tossing are space-like separated events $-h^{\prime} L_{h}^{e} h^{\prime \prime}$ and the counterfactual comes out true at $e, h$.

The theoretical verdict proclaiming the counterfactual to be false comes about as follows: The closest history at which the antecedent is true ("I bet on tails") is one that branches off from the actual history at the actual betting event. The tossing is after this, and in the history in which $U$ bet on tails as well as in the actual history, the toss is an indeterministic event with two possible outcomes. None of these is preferred in any way (even though in the actual history, the coin did show tails). Thus, it is not the case that in all the closest histories in which $U$ bet on tails, the coin showed tails. 
Against this analysis, the intuitive verdict holds that since the betting and the tossing are independent, in the closest history with $U$ betting on tails, the coin should still have shown tails, as it actually did.

In the framework of branching space-times there is a formally precise notion of such independence, namely space-like relation. While it is true that in actual betting cases, space-like relatedness of betting and tossing can hardly be realized, ${ }^{22}$ a model that pictures these two events as space-like related correctly reproduces the way we intuitively think about the setup. Figure 4 shows the difference between the two types of model. If betting and tossing are pictured as space-like related, the counterfactual (6) comes out true: history $h^{\prime}$ ( $U$ betting on tails, coin showing tails as it actually did) is closer to the actual history than history $h^{\prime \prime}$, and at $e^{\prime}, h^{\prime}$, the consequent of the counterfactual is true.

\subsection{Fragility}

In branching space-times, a given event $e \in W$ cannot happen at a different space-time location, nor in a manner different from the way it actually did. To make things worse, if an event occurs, its whole past is fixed uniquely. In the terminology of Lewis $(1986,196)$, BST events are fragile. Fragility is usually considered problematic. Suppose that I am having a cup of coffee now, and an hour ago, in Australia a certain person in fact waved her arm (this takes place in the causal past, or backward light cone, of my having my coffee). Suppose that the person could have refrained from waving her arm. Through interference with some solar neutrinos, say, this might have altered the trajectory of one of the water molecules in my cup. Accordingly, if the person in Australia hadn't waved her arm, I could not have had my coffee the way I did; the actual event of my having my coffee would not have occurred. ${ }^{23}$ This sounds weird. ${ }^{24}$

In pure BST we can only talk about events occurring or not occurring, so the above verdict may seem unavoidable. However, there are two options for specifying events in a non-fragile way. First, one can specify so-called disjunctive events, which are sets of point events not overlapped by a single history. The disjunctive event occurs iff one of its members occurs, i.e., it can occur in many ways (cf. Belnap (2005) for details). Thus, in the coffee case, one may take the event of having one's coffee to be disjunctive and argue that the particular (disjunctive) event of my having my morning coffee would still occur, no matter whether the Australian waved her arm or not. While this avoids the fragility problem, one may wonder how one should justify the particular set that is used in specifying the disjunctive event. The 
second option therefore uses the properties that were added to pure BST (cf. section 3.2 above) in order to provide a justification. Relative to a given description in terms of some properties, the actual event and many once-possible events will count as "the same thing" - arguably, no description can be exhaustive. E.g., relative to an everyday description, I would still be having my morning coffee in the same particular way, no matter whether the coffee was a little warmer or colder, whether I was drinking from this or from another mug, or whether I was sitting differently, etc. The Australian's waving her arm will obviously not make any difference to the event so conceived.

\subsection{Late Departure: The COAT Story}

Our definition of comparative closeness subscribes to a principle that is known as "late departure" $: 25$ histories are the closer the later they branch. This principle is operative in other treatments of counterfactuals as well, cf., e.g., Lewis (1986, 47f., 58ff.) and Bennett (2003, 220). The principle faces the following notorious objection: ${ }^{26}$

My coat was not stolen from the restaurant where I left it. There were two chances for theft [...]. They would have involved different potential thieves: and the candidate for the later theft is a rogue who always sells his stuff to a pawnbroker named Fence.

If the closest A-world involves the latest admissible fork, it follows from the above story that if my coat had been stolen from the restaurant, it would now be in Fence's shop. That is not acceptable.

(Bennett 2003, 219f.; emphasis in original)

We have to confess that our treatment leads to the same verdict. In line with Bennett and others, we agree that in some cases, an intuitively acceptable comparative closeness ordering cannot be based on spatio-temporal considerations alone, but it seems that this phenomenon escapes formal treatment.

\subsection{A REMARK ON THE "ANALYTiCAL CIRClE"}

What sort of elucidation can we hope to achieve through an analysis of the truth conditions of counterfactuals? As Lewis $(1986,43)$ has conceded, his analysis has to employ what may be called an "analytical circle": from intuitively known truth values of counterfactuals to the assessment of similarity back to the truth value of counterfactuals. This circularity is a ubiquitous feature of philosophical analysis, and our analysis is not completely free from it either. However, it is an advantage of our analysis that the circle has at least become larger. We derive the truth value of counterfactuals from verdicts about similarity, but 
these are based not on counterfactual statements, but on the appraisal of what was historically possible. In some cases, we will know what was historically possible only through the truth of some counterfactuals, but there are other, independent sources too, like generalizations from experiments or symmetry considerations.

\section{Summary}

We have argued that while no all-encompassing, rigorous analysis of counterfactuals seems to be forthcoming, rigor is possible for the class of historical counterfactuals that plays an important role in everyday arguments as well as in scientific contexts. Historical counterfactuals are based on historical possibility. We elucidated both the notion of historical possibility and the notion of historical counterfactuals in the framework of branching space-times. Following the lead of many other analyses of counterfactuals, the semantics was based on a similarity relation, here called "comparative closeness". This notion could be defined fully rigorously in the branching space-times framework, consequently allowing for more or less standard semantic clauses that are no longer vague.

By widening the "analytical circle", our analysis is not just formally more rigorous than existing treatments of counterfactuals, but also philosophically more dependable.

\section{Acknowledgements}

We would like to thank Nuel Belnap and Geert Keil for helpful comments on an earlier draft of this paper. We also thank audiences at Bonn and at Kraków for comments on related talks, and two anonymous referees for their helpful comments. Financial support by the State's Committee of Research (KBN), grant no. 2H01A 023 25, is acknowledged.

\section{Appendix}

\section{A. Belnap's analysis of counterfactuals in "pure event" branching space-times}

Nuel Belnap (2005) proposed an alternative analysis of counterfactuals, which is carried out in basic branching space-times only. Since

cfsynth_revised_18mar05.tex; 20/04/2005; 12:42; p.19 
he links true counterfactual-like statements to facts about originating causes of corresponding events, there is an affinity between historical counterfactuals and statements amenable to this analysis.

The language of basic BST is austere, fucussing on occurrence propositions, i.e., propositions stating that a given event occurs. In BST there are a few kinds of events. In what follows we need, apart from a point event, an outcome chain $O$, defined as a non-empty and lower bounded chain of point events from $W$.

In the spirit of possible-worlds theories, occurrence propositions are identified with appropriate sets of histories. The occurrence proposition for a point event $e$ is the set $H_{(e)}=\{h \in H i s t \mid e \in h\}$. The occurrence proposition for an outcome chain $O$ is $H_{\langle O\rangle}=\{h \in H i s t \mid O \cap h \neq \emptyset\}$. We say that a proposition is true in $h$ iff $h$ belongs to this proposition.

To explain some auxiliary notions, we write $e<O$ iff $\forall x(x \in O \rightarrow$ $e<x) . g \perp_{e} h$ means that $e$ is a choice point for histories $g$ and $h$. For $H$ a set of histories, $h \perp_{e} H \stackrel{d f}{\leftrightarrow} \forall h_{2}\left(h_{2} \in H \rightarrow h \perp_{e} h_{2}\right)$. For undividedness $\equiv_{e}$ of Definition 3 and $H$ a set of histories, $h \equiv_{e} H \stackrel{d f}{\leftrightarrow}$ $\forall h_{2}\left(h_{2} \in H \rightarrow h \equiv_{e} h_{2}\right)$.

One of Belnap's aims in his (2005) is to identify what he calls originating causes, or causae causantes, of a given outcome chain $O$. He first singles out choice points in the past of $O$ at which histories that avoid $O$ could be 'selected'. He calls the set of such choice points past causal loci for $O$, defined as: $p c l(O)=\left\{e \mid e<O\right.$ and $\left.\exists h h \perp_{e} H_{\langle O\rangle}\right\}$. For each $e$, the relation $\equiv_{e}$ of undividedness at $e$ determines a partition $\Pi_{e}$ of the set $H_{(e)}$. Given $e<O$, here is a single element of $\Pi_{e}$ containing some histories that intersect non-emptily with $O$, that is, histories in which $O$ occurs. This single element, $\Pi_{e}\langle O\rangle$, is called the projection of $O$ onto $e$, and is defined as: $\Pi_{e}\langle O\rangle \stackrel{d f}{=}\left\{h \in H_{(e)} \mid h \equiv_{e} H_{\langle O\rangle}\right\}$. The pair consisting of a past causal locus $e$ for $O$ and the projection $\Pi_{e}\langle O\rangle$ of $O$ onto $e$ is written $e \longmapsto \Pi_{e}\langle O\rangle$; it is called a basic transition. Basic transitions $e \longmapsto \Pi_{e}\langle O\rangle$, where $e$ is a $p c l$ for $O$, are identified with originating causes of $O$.

What is then the occurrence proposition for transition $e \longmapsto \Pi_{e}\langle O\rangle$ ? Belnap argues for the 'material implication' sense of this proposition, that is, the following: The occurrence proposition for $e \longmapsto \Pi_{e}\langle O\rangle$ is true in history $h$ iff if $h \in H_{(e)}$, then $h \in \Pi_{e}\langle O\rangle$. By the definition of 'material implication', this means that $e \longmapsto O$ occurs in $h$ iff $e \notin h$ or $h \in \Pi_{e}\langle O\rangle$.- Given this sense of occurrence propositions for transitions, it follows that a basic transition $e \longmapsto \Pi_{e}\langle O\rangle$ is an inns condition for $O$, i.e., an insufficient but non-redundant part of a necessary and sufficient condition for $O$. 
Having introduced the machinery, we may now take up Belnap's (2005) account of counterfactuals in pure BST. He agrees with Lewis and others in pointing out a strong link between counterfactual conditionals and strict conditionals. E.g., Lewis (1973) asserts this link and goes on to argue that counterfactuals need to be interpreted as variably strict conditionals, where the variability is guided by some similarity considerations. Belnap denies that there can be an objective theory of the necessary variability.

He first shows that some important subjunctive conditionals may be given a BST reading as simple strict conditionals without any variability. Consider an outcome event $O$ and one of its causae causantes, $e \longmapsto \Pi_{e}\langle O\rangle$. It follows straight from the definitions of "inns condition" that if $e \longmapsto \Pi_{e}\langle O\rangle$ hadn't occurred, $O$ wouldn't have occurred. There is no need for an elaborate account of counterfactuals here. We are even entitled to conclude that ... O wouldn't have occurred, but some other alternative $\Pi_{e}\langle h\rangle$ would have occurred, just on the basis of the "material implication" reading of the occurrence proposition for $e \longmapsto \Pi_{e}\langle O\rangle$.

In a second step, Belnap allows for a notion of similarity to enter the picture: It makes (objective) sense to grade the alternatives in $H_{(e)}$ with respect to their similarity to actuality (i.e., $O$ occurring) by considering the other originating causes of $O$ as well. Thus, a history in which $e \longmapsto \Pi_{e}\langle O\rangle$ is the only originating cause of $O$ that fails is in some good sense closer to actuality than a history in which two or more of the originating causes fail. ${ }^{27}$ Still, one arrives at a strict conditional reading of counterfactuals that Belnap takes to be only slightly more illuminating than the first one. In the end, his advice is to exclude counterfactuals from objective theories, reserving their use to "sportscasters and military historians" (R.K. Meyer). In their place, objective theories should only rely on explicitly formulated strict conditionals.

We fully agree with Belnap's verdict as far as pure BST is concerned. However, even pure BST has the resources to define similarity orderings ("comparative closeness") that are more general than counting causae causantes. We have shown that based on two slight extensions of BST, these orderings can be employed to give rigorously defined truth conditions for informative counterfactuals. Thus, pace Belnap, there seems to be room for counterfactuals in objective theories.

\section{Notes}

${ }^{1}$ Stalnaker (1968) uses $A>C$ for the same purpose.

2 "Iff" stands for "if and only if". 
${ }^{3}$ Historically, the first analysis of counterfactuals by Goodman (1947) followed a different guiding idea. Bennett (2003) labels Goodman's approach "support theory", to be distinguished from the "world theory" approach of Stalnaker, Lewis, and others, which is the focus of this paper. Roughly, in a support theory, $A \square \longrightarrow C$ is taken to be true iff there is a true proposition $S$ ("support") and a law-statement $L$ such that $A \& S \& L$ implies $C$. Certainly not any $S$ qualifies - take $\neg A$, and all counterfactuals come out true. Thus, Goodman and his followers require $S$ to be cotenable with $A$, which notion requires further analysis. There is quite some disagreement as to whether Goodman's approach can be made to work. If so, that would facilitate the analysis of causal independence (cf., e.g., example sentence (6) below). However, we agree with Bennett, whose careful analysis of the Goodman approach (Bennett 2003, Chap. 20 and $\S 129$ ) shows convincingly that support theories face insurmountable difficulties. Thus, we continue our discussion solely in the "world theory" framework.

${ }^{4}$ What we call "historical possibilities" may also be called "real possibilities"; cf. Lewis $(1999,423)$ and Xu's (1997) phrase "possibilities based on reality".

${ }^{5}$ If you are not convinced by this "large scale" example, use your favorite quantum mechanical example instead.

${ }^{6}$ For a critical discussion of determinism and counterfactuals cf. Kvart (1986, 178f., 256ff.), who also strongly favors indeterminism.

${ }^{7}$ Maybe Lewis believed that he had to hold on to determinism because he thought that there was no well worked-out theory of localized indeterminism-his letter, quoted in Bennett $(2003,204)$, might suggest this. However, as we will show, branching space-times provides the necessary resources.

8 This will normally be the case, but not, e.g., if it was a trick coin whose two sides both show tails. It depends on the coin that was actually used.

${ }^{9}$ Difficult questions of identity criteria for individuals are involved. We assume that at least for precious stones, the kind of stone constitutes part of its identity, but maybe not, e.g., the cutting, and certainly not the question of whether there is some dirt on its surface.

${ }^{10}$ As we remarked in the previous note, difficult questions, e.g., regarding identity or generalizations, may be involved, but these questions arise already independently of the analysis of counterfactuals.

${ }^{11}$ Note that in the technical sense of the theory of branching space-times to be explained below, what is given by $e$ is not a single history, but rather an initial segment of a possibly large bundle of histories (which may diverge after $e$ ).

${ }^{12}$ For Belnap's postulates, cf. his (1992) paper; for a discussion and an alternative set of postulates, cf. Placek (2003).

13 Cf. Müller (2002).

${ }^{14}$ Note that not all models of BST permit locations in the above sense. For an illustration, cf. Müller 2005.

15 Appendix A shows that BST alone is too austere a theory to permit informative counterfactuals.

${ }^{16}$ A similar intuition underlies the analysis of conditionals given by Thomason and Gupta (1980). Our approach is both narrower, aiming only at a subclass of counterfactuals and not at conditionals in general, and wider, incorporating the spatial aspect. The latter allows us to escape some of the complications of the Thomason/Gupta-analysis related to causal coherence. Furthermore, BST makes our analysis applicable to a relativistic setting.-Arguably, this intuition has problematic consequences. Cf. Section 6.3 below for discussion.

cfsynth_revised_18mar05.tex; 20/04/2005; 12:42; p.22 
${ }^{17}$ Lewis (1973) based the semantics for counterfactuals on a family of weak orderings. He extended his analysis to families of partial orderings in his (1981), which is the semantics that we will be using below.

18 Cf. the definition of 'weak similarity' in Placek (2005) and Müller (2002).

19 The best description of that framework known to us is given in Chap. 8 of Belnap et al. (2001).

${ }^{20}$ Cf. note 13 above.

${ }^{21}$ Bennett (2003, 234f.), who attributes the example to Tichý, describes some of the controversy surrounding it.

${ }^{22}$ On a typical distance between toss and bet of, say, 1 meter, this would mean that betting and tossing have to occur within less than $3 \cdot 10^{-9}$ seconds. Such margins of precision have, however, been realized in some quantum-mechanical experiments: cf., e.g., Weihs et al. (1998).

${ }^{23}$ According to Lewis's project of a counterfactual analysis of singular causation, the truth of this counterfactual would mean that the Australian's arm-waving causes my drinking my coffee, which is obviously absurd.

${ }^{24}$ In branching space-times, the fragility is even more extreme, since a mere difference of the causal past, without any physical interaction, will already differentiate events.

${ }^{25}$ Cf. Mårtensson (2000, 79ff.).

26 The example is Pollock's; cf. Nute $(1980,104)$. We follow the careful wording of Bennett (2003, 219f.), which is immune against the charge that a backtracking conditional is involved.

27 Since the spatiotemporal ordering plays no role for this notion of closeness, the charge against "late departure" discussed in section 6.3 does not apply.

\section{References}

Barker, S. (2003). 'A dilemma for the counterfactual analysis of causation'. Australasian Journal of Philosophy 81:62-77.

Belnap, N. (1992). 'Branching space-time'. Synthese 92:385-434.

Belnap, N., Perloff, M. and Xu, M. (2001). Facing the Future. Oxford: Oxford University Press.

Belnap, N. (2005). 'A theory of causation: causae causantes (Originating causes) as inus conditions in branching space-times'. British Journal for the Philosophy of Science (forthcoming).

Bennett, J. (2003). A Philosophical Guide to Conditionals. Oxford: Oxford University Press.

Edgington, D. (1995). 'On conditionals'. Mind 104:235-329.

Edgington, D. (2004). 'Counterfactuals and the benefit of hindsight'. In P. Dowe and P. Noordhof (eds.), Causation and Counterfactuals. London: Routledge.

Goodman, N. (1947). 'The problem of counterfactual conditionals'. Included as part of his Fact, Fiction and Forecast, London: Athlone Press 1954.

Kvart, I. (1986). A Theory of Counterfactuals. Indianapolis, IN: Hackett.

Lewis, D. (1973). Counterfactuals. Oxford: Blackwell.

Lewis, D. (1981). 'Ordering semantics and premise semantics for counterfactuals'. Journal of Philosophical Logic 10:217-234.

Lewis, D. (1986). Philosophical Papers, Vol. II. Oxford: Oxford University Press.

cfsynth_revised_18mar05.tex; 20/04/2005; 12:42; p.23 
Lewis, D. (1999). Papers in Metaphysics and Epistemology. Cambridge: Cambridge University Press.

Mårtensson, J. (2000). Subjunctive Conditionals and Time. Göteborg: Acta Universitatis Gothoburgensis.

Müller, T. (2002). 'Branching space-time, modal logic, and the counterfactual conditional'. In Placek, T. and J. Butterfield (eds.), Non-locality and Modality. Dordrecht: Kluwer 2002, 273-291.

Müller, T. (2005). 'Probability theory and causation. A branching space-times analysis'. British Journal for the Philosophy of Science (forthcoming).

Nute, D. (1980). Topics in Conditional Logic. Dordrecht: Reidel.

Placek, T. (2003). 'On Belnap's branching space-times'. In Hintikka J., T. Czarnecki, K. Kijania-Placek, T. Placek and A. Rojszczak (eds.), Philosophy and Logic. In Search of the Polish Tradition. Dordrecht: Kluwer 2003, 77-92.

Placek, T. (2005). 'Comparative similarity in branching space-times'. International Journal of Theoretical Physics (forthcoming).

Stalnaker, R. (1968). 'A theory of conditionals'. In Studies in Logical Theory, American Philosophical Quarterly Monograph Series, 2. Oxford: Blackwell 1968, 98-112.

Thomason, R. and A. Gupta (1980). 'A theory of conditionals in the context of branching time'. In W. Harper, R. Stalnaker and G. Pearce, Ifs. Dordrecht: Reidel 1980, 299-322.

Xu, M. (1997). 'Causation in branching time (I): Transitions, events and causes'. Synthese 112:137-192.

Weihs, G., Jennwein, T., Simon, C., Weinfurter, H. and Zeilinger, A. (1998). 'Violation of Bell's inequality under strict Einstein locality conditions'. Physical Review Letters 81:5039-5043.

cfsynth_revised_18mar05.tex; 20/04/2005; 12:42; p.24 